\title{
Formoterol Fumarate/Roxithromycin
}

National Cancer Institute

\section{Source}

National Cancer Institute. Formoterol Fumarate/Roxithromycin. NCI Thesaurus. Code C71161.

A combination preparation of the fumarate salt of a beta2-adrenergic receptor agonist and a macrolide antibiotic, with muscle-sparing and anti-cachexia effects. Formoterol appears to antagonize cachexia by reducing proteolysis mediated through the ubiquitinprotease pathway. Roxithromycin strongly inhibits inflammatory cytokine production and secretion from $T$ cells and macrophages in vitro and in vivo. The combination exhibits a greater muscle sparing effect than either drug given individually at comparable doses. 